\title{
Criminologie
}

\section{Les stratégies de recherche d'aide des femmes victimes de violence conjugale : le cas des femmes référées aux CLSC par les policiers}

\author{
Maryse Rinfret-Raynor, Solange Cantin et Lise Fortin
}

Volume 30, numéro 2, automne 1997

Violences familiales

URI : https://id.erudit.org/iderudit/017406ar

DOI : https://doi.org/10.7202/017406ar

Aller au sommaire du numéro

Éditeur(s)

Les Presses de l'Université de Montréal

ISSN

0316-0041 (imprimé)

1492-1367 (numérique)

Découvrir la revue

Citer cet article

Rinfret-Raynor, M., Cantin, S. \& Fortin, L. (1997). Les stratégies de recherche d'aide des femmes victimes de violence conjugale : le cas des femmes référées aux CLSC par les policiers. Criminologie, 30(2), 87-108.

https://doi.org/10.7202/017406ar
Résumé de l'article

This article deals with formal and informal resources used by women victims of conjugal violence in their search for help. It explores the type of resources used and their relationship with a number of factors such as the socio-economic characteristics of the women and, the nature and duration of the violence which they have suffered. Prior to reporting on the results of a specific case of formal resources utilization, this article presents a review of the literature on the process of seeking help, then it focuses on the major role played by the social support network. Also, it reviews the major studies dealing with the strategies used by women victims of conjugal violence in obtaining help. Finally, the article reports on the main results of a study conducted in the context of a pilot project to bring help to victims of conjugal violence by the police and the Local Community Services Centers (CLSC) of the Montreal Urban Community. This pilot project started in 1990 has since been extended to the entire territory covered by the Montreal Urban Community. The results of this study show that the majority of the women referred by the police officers did receive help at the CLSC. However, only one third of the women referred did meet with a social practioner following the referral. Also, it was observed that when the period between the referral and the first contact by the social practitioner was short, the women more easily accepted to meet with a worker from the CLSC. A third of the women referred already had a file with the CLSC, which indicated that the violence problem was known in one case out of four. Meeting with a CLSC worker was found to provide a greater diversity in the type of help received.
Ce document est protégé par la loi sur le droit d'auteur. L'utilisation des services d’Érudit (y compris la reproduction) est assujettie à sa politique d'utilisation que vous pouvez consulter en ligne.

https://apropos.erudit.org/fr/usagers/politique-dutilisation/ 


\section{LES STRATÉGIES DE RECHERCHE D'AIDE DES FEMMES VICTIMES DE VIOLENCE CONJUGALE : LE CAS DES FEMMES RÉFÉRÉES AUX CLSC PAR LES POLICIERS Maryse Rinfret-Raynor', Solange Cantin ${ }^{2}$ et Lise Fortin}

This article deals with formal and informal resources used by women victims of conjugal violence in their search for help. It explores the type of resources used and their relationship with a number of factors such as the socio-economic characteristics of the women and, the nature and duration of the violence which they have suffered. Prior to reporting on the results of a specific case of formal resources utilization, this article presents a review of the literature on the process of seeking help, then it focuses on the major role played by the social support network. Also, it reviews the major studies dealing with the strategies used by women victims of conjugal violence in obtaining help. Finally, the article reports on the main results of a study conducted in the context of a pilot project to bring help to victims of conjugal violence by the police and the Local Community Services Centers (CLSC) of the Montreal Urban Community. This pilot project started in 1990 has since been extended to the entire territory covered by the Montreal Urban Community. The results of this study show that the majority of the women referred by the police officers did receive help at the CLSC. However, only one third of the women referred did meet with a social practioner following the referral. Also, it was observed that when the period between the referral and the first contact by the social practitioner was short, the women more easily accepted to meet with a worker from the CLSC. A third of the women referred

1. Professeur titulaire à l'École de service social, vice-doyenne aux études à la Faculté des arts et des sciences de l'Université de Montréal et co-directrice du Centre de recherche interdisciplinaire sur la violence familiale et la violence faite aux femmes (CRI-VIFF), Université de Montréal, C.P. 6128, Succursale Centre-Ville, Montréal, Qc, H3C $3 \mathrm{~J} 7$.

2. Coordonnatrice de l'equipe VICTOIRE (Violence conjugale : transformer et orienter par l'intervention et la recherche), Centre de recherche interdisciplinaire sur la violence familiale et la violence faite aux femmes (CRI-VIFF), Université de Montréal, C.P. 6128, Succursale Centre-Ville, Montréal, Qc, H3C 3J7.

3. Etudiante au Ph.D. en sciences hurnaines appliquées. 
already had a file with the CLSC, which indicated that the violence problem was known in one case out of four. Meeting with a CLSC worker was found to provide a greater diversity in the type of help received.

Depuis que la violence conjugale est considérée comme un problème social, les services publics et communautaires sont interpellés pour mieux répondre aux besoins des victimes et intervenir efficacement auprès des agresseurs. On exige d'eux des efforts de coordination et de concertation qui ont peu de précédent dans d'autres problématiques sociales. Ainsi, le gouvernement du Québec lançait en 1995 une politique interministérielle en matière de violence conjugale. Son message central est que les solutions au problème doivent « [...] être conjointes, complémentaires et étroitement liées » (Gouvernement du Québec, 1995, p. 5). Par ailleurs, les milieux d'intervention sont souvent déroutés devant l'hésitation des femmes à révéler la violence qu'elles subissent, leur refus de l'aide offerte ou leur abandon du processus d'aide amorcé. Pour maintenir la volonté d'offrir une aide appropriée, il est donc essentiel de comprendre la dynamique de recherche d'aide des victimes de violence conjugale.

Cet article se penche sur l'utilisation des ressources par les femmes victimes de violence conjugale. Il aborde en particulier la question du type de ressources utilisées et des liens avec certains facteurs (caractéristiques socio-économiques des femmes, nature et durée de la violence subie, ressources utilisées antérieurement). Avant de présenter un cas particulier d'utilisation de ressources formelles, cet article résume, dans une première partie, l'état des connaissances sur la notion de recherche d'aide, sur le rôle important joué par le réseau de soutien social et sur les stratégies de recherche d'aide des femmes victimes de violence conjugale. La deuxième partie décrit les principaux constats d'une étude menée à l'intérieur d'un projet que le Service de police de la Communauté urbaine de Montréal et des Centres locaux de services communautaires (CLSC) ont mis sur pied en 1990 à titre de projet pilote et qui s'est depuis étendu à tout le territoire de la Communauté urbaine de Montréal. Cette étude contient peu d'informations sur l'utilisation des autres ressources par les femmes violentées. Il est cependant apparu essentiel de la situer dans un cadre conceptuel plus large abordant la notion générale de recherche d'aide et l'ensemble des stratégies de recherche d'aide des femmes victimes de violence conjugale. Cela évitera de considérer comme des particularités propres aux femmes violentées ce qui peut en fait constituer des caractéristiques communes à la recherche d'aide face à divers types de difficultés. 


\section{LA NOTION DE RECHERCHE D'AIDE}

Rechercher de l'aide est une des options dont l'individu dispose pour faire face à ses difficultés. Les premières études sur la recherche d'aide se sont intéressées à l'usage de l'aide formelle dans le champ de la santé, en particulier de la santé mentale (Gurin et al., 1960; Kadushin, 1969). Les études plus récentes concernent toujours en grande partie ces mêmes champs d'intervention (Bland et al., 1990 ; Greenley et Mullen, 1990 ; Pescosolido, 1992).

Certains auteurs ont tenté de đégager les étapes du processus de recherche d'aide. DePaulo (1982) a identifié cinq étapes communes à plusieurs modèles : déclenchement du processus par la reconnaissance du besoin d'aide; décision de rechercher de l'aide ou non ; sélection d'une source d'aide appropriée ; initiation et exécution de la demande d'aide ; réaction à la réponse de l'aidant. Ces étapes ne sont pas nécessairement linéaires et peuvent être influencées par la disponibilité d'une aide acceptable ou par les réactions d'aidants antérieurs.

\section{Rôle important du réseau de soutien social}

Les études sont unanimes à constater que la recherche d'aide chez les professionnels survient généralement après de nombreuses démarches auprès d'amis, de membres de la famille et de ressources communautaires (Gourash, 1978 ; Kadushin, 1969). Ces sources d'aide informelle sont disponibles a l'intérieur du réseau social de la personne.

Moos et Mitchell (1982) ont défini quatre catégories de facteurs qui influencent les réseaux : les facteurs environnementaux, les caractéristiques socio-démographiques et personnelles, les événements stressants et les mécanismes individuels de réponse.

Les facteurs environnementaux englobent les caractéristiques physiques et architecturales, organisationnelles, suprapersonnelles et sociales dont l'influence s'exerce à travers des processus. Par exemple, les caractéristiques physiques ou organisationnelles des voisinages facilitent ou restreignent les possibilités de se rencontrer et d'interagir. Des variables telles que l'âge, le revenu, l'éducation et le statut conjugal influencent l'accès et la participation à des contextes sociaux, ce qui a des conséquences sur la création des réseaux. Par exemple, Depner et Ingersoll ( 1980 ; cités par Moos et Mitchell, 1982) montrent que les femmes mariées sont la principale source de soutien émotionnel pour leur conjoint, alors qu'elles-mêmes en reçoivent autant de leurs parents et amis que du conjoint lui-même. Les ressources personnelles telles que l'estime de soi et les compétences sociales peuvent influencer le développement du réseau. Plusieurs événements stressants (deuil, 
déménagement, perte d'emploi, etc.) perturbent les liens établis et limitent l'accès aux sources habituelles de soutien (Schaefer et al., cités par Moos et Mitchell, 1982). Des tensions chroniques telles que les soucis financiers peuvent mener à une diminution de l'habileté et de la motivation à maintenir des liens sociaux (Pearlin et Lieberman, 1979 ; cités par Moos et Mitchell, 1982). Par contre, d'autres événements stressants peuvent provoquer une mobilisation du soutien dans le réseau (naissance d'un enfant, par exemple).

L'aide informelle dispensée par les proches, les amis et le voisinage jouerait de plus un rôle crucial de filtrage et de référence dans l'accès aux ressources formelles. Dans une étude portant sur 2500 hommes et femmes, Warren (1981) a observé que seulement $2 \%$ des personnes nécessitant de l'aide accèdent directement aux ressources d'aide formelle, sans l'intermédiaire du réseau informel. Les individus utilisent très souvent plusieurs sources d'aide simultanément pour un même problème.

\section{Typologie des problèmes et recherche d'aide}

Warren (1981) a élaboré une typologie des problèmes individuels selon qu'ils se réferrent à un degré faible, moyen ou élevé d'expertise professionnelle.

En ce qui concerne les problèmes liés à un faible degré d'expertise, il n'existe pas de consensus quant à leur définition ni à l'application d'une expertise particulière. Le caractère non uniforme du problème limite la valeur d'une action formelle concertée. Les tensions entre parents et enfants, le budget familial, et l'adaptation après l'arrivée d'un bébé, par exemple, entrent dans cette catégorie.

Pour les problèmes se rapportant à un degré d'expertise moyen tels que l'alcoolisme, l'obésité et les difficultés conjugales, la technologie est en đéveloppement, et plusieurs approches concurrentes proposent des solutions dont l'efficacité est limitée. Les querelles entre professions pour s'approprier des juridictions sur le problème sont fréquentes. De même, des conflits surviennent entre les secteurs formel et informel quant à leur efficacité respective vis-à-vis du problème.

Enfin, les problèmes se rapportant à un niveau d'expertise élevé ont une technologie bien définie et sophistiquée, souvent limitée à un champ hautement spécialisé. Il existe un consensus sur le fait que la situation en cause constitue vraiment un problème. Les actions des aidants non experts ne peuvent pas vraiment en changer la nature ou proposer des solutions. On compte dans cette catégorie l'hypertension artérielle, la schizophrénie, le dépistage du cancer, les soins prénataux, etc. 


\section{Autres déterminants de la recherche d'aide}

Parmi les éléments qui militent pour le choix de l'aide informelle, Wills (1982) note son aspect familier, son accessibilité géographique et sa gratuité. Le soutien social permet à l'individu de maintenir son estime de lui-même (Gourash, 1978). L'aide informelle s'insère dans un contexte d'échanges réciproques qui minimise l'impression d'être redevable ou de s'imposer (Fisher et Nadler, 1982).

À l'inverse, la perspective d'être évalué négativement par les membres de la communauté ou par d'autres types d'aidants inhibe la recherche d'aide (Veroff et al., 1981). DePaulo et Fisher (1980) ont montré que les gens attribuent effectivement de plus faibles habiletés aux personnes qui reçoivent de l'aide, surtout si elles ont dû la demander. Le fait de rechercher de l'aide peut constituer une menace à l'estime de soi puisque, pour plusieurs, cela signifie admettre ouvertement son inadaptation personnelle (Homans, 1961 ; cité par DePaulo, 1982). La peur du refus et celle d'être perçu comme un fardeau (Kalish, 1967 ; cité par DePaulo, 1982), le fait de devoir divulguer des informations négatives à son sujet et la peur de ce que l'on peut découvrir en demandant de l'aide (Snyder et al., 1982 ; cités par DePaulo, 1982) constituent d'autres facteurs qui inhibent la recherche d'aide.

\section{LA RECHERCHE D'AIDE DES FEMMES VIOLENTÉES}

L'enquête de Statistique Canada sur la violence envers les femmes (Rodgers, 1994) montre que les femmes violentées par leur conjoint se tournent surtout vers des membres de leur famille ( $45 \%$ ) et vers leurs amis et voisins (44\%). Cette enquête révèle que $26 \%$ seulement des voies de fait contre la conjointe ont été signalés à la police. Au total, $24 \%$ des femmes victimes de violence conjugale ont déclaré avoir fait appel à un ou plusieurs services sociaux, incluant les maisons d'hébergement. Près du quart (23\%) en ont parlé à un médecin et $7 \%$ à un membre du clergé. Cette réalité rejoint les constatations selon lesquelles le réseau social informel est beaucoup plus utilisé que les ressources formelles pour obtenir de l'aide. L'enquête tend de plus à confirmer la tendance souvent observé à l'effet que l'utilisation d'une ressource formelle est associée au recours à une ou plusieurs autres ressources : ainsi, les femmes qui ont fait appel aux policiers ont été beaucoup plus nombreuses à avoir recours à un ou plusieurs services sociaux (46\%) que celles qui n'avaient rien signalé à la police (17\%).

Par contre, des comparaisons entre les femmes qui ne vivent pas de violence et les femmes violentées montrent que ces dernières utilisent davantage les ressources formelles. Ainsi, l'enquête exploratoire de Santé Québec sur la violence envers la conjointe révèle que les femmes qui ont eu 
recours aux services de santé et celles qui ont consulté un médecin généraliste au cours des deux semaines précédant l'enquête ont davantage subi de la violence physique que celles qui n'avaient pas consulté (Riou et al., 1996). Ces données confirment les observations faites par Chénard et al. (1990).

Nous devons donc conclure que les femmes violentées font largement appel aux ressources socio-sanitaires sans nécessairement dénoncer la violence subie. Ce qui peut avoir pour conséquence une intervention souvent non appropriée et même dangereuse puisqu'elle ne tient pas compte de l'origine réelle de nombreux problèmes (Rinfret-Raynor et Turgeon, 1995).

\section{Les femmes victimes de violence et le soutien social}

Étant donné le rôle critique du soutien social dans la recherche d'aide, il n'est pas surprenant de retrouver l'isolement social des femmes violentées au cœur de la problématique de recherche d'aide de ces femmes. Ainsi, plus de $50 \%$ des 42 femmes interrogées par Pahl (1985) n'avaient aucune ou seulement une seule personne vers qui se tourner pour demander de l'aide. L'enquête exploratoire de Santé Québec montre que les femmes plus isolées socialement sont beaucoup plus nombreuses à subir de la violence verbale ou physique de la part de leur conjoint (Riou et al., 1996). L'enquête de Statistique Canada a montré que $22 \%$ des femmes victimes de violence n'en avaient parlé à personne auparavant (Rodgers, 1994).

Les résultats d'une étude de Mitchell et Hodson (1983) suggèrent que l'influence négative de la violence sur la santé psychologique et l'adaptation s'exerce en partie à travers les effets nuisibles de la violence sur le soutien social. Lorsque la violence s' intensifie, les amis sont moins susceptibles de répondre adéquatement aux demandes d'aide des femmes. Cette recherche a aussi démontré qu'un plus grand nombre de contacts de la femme violentée avec des amis ou des membres de la famille, sans la présence de son conjoint, est significativement associé à un plus grand nombre d'aidants potentiels, à des réponses plus empathiques de la part des amis et à moins de comportements d'évitement à l'égard de la violence.

\section{Les autres obstacles à la recherche d'aide des femmes victimes de violence}

Le sentiment de honte et de culpabilité contribue à la réticence des femmes à créer et à maintenir des contacts d' aide. La violence est souvent pour la femme un indicateur d'échec personnel. Lorsque cette violence est dévoilée au grand jour, cet échec se traduit en « stigma public » (Dobash et Dobash, 1985). 
La théorie de l'impuissance apprise développée par Seligman (1975; cité par Hotaling et al., 1988) a été appliquée aux femmes victimes de violence conjugale par Walker (1979) qui soutient que celles-ci présentent des signes «d'impuissance apprise » pouvant diminuer leur estime d'elles-mêmes et leur habileté à prendre les moyens nécessaires pour modifier leur situation. Freeman (1979) souligne que les femmes « prises au piège dans leur mariage » peuvent ne pas avoir l'habitude de décider ni la force et la confiance nécessaires pour entreprendre des démarches constructives. Elle note de plus une méconnaissance du type d'aide approprié à leurs problèmes et de l'attitude appropriée en période de crise.

Certaines femmes craignent de perdre la garde des enfants, dépendants du père sur le plan économique. Les menaces de représailles de la part du conjoint et le confinement à la maison par la force sont d'autres facteurs qui empêchent la recherche d'aide (Ford, 1983 ; Dobash et Dobash, 1985).

Plusieurs fermmes vivent des conditions personnelles et sociales qui ajoutent des difficultés particulières à celles de l'ensemble des femmes. C'est le cas notamment des femmes immigrantes ou membres d'un groupe ethnique minoritaire, des femmes vivant avec une ou des limitations fonctionnelles, et des femmes âgées.

\section{La réponse des institutions}

Les écrits sur la réponse des institutions aux demandes d'aide des femmes violentées soulignent plusieurs lacunes : le manque de compréhension du phénomène de la violence conjugale (Gelles, 1976); l'absence d'alternatives viables pour les femmes victimes (Roy, 1977); la nécessité pour ces femmes de consulter plusieurs organismes avant d'obtenir toute l'aide nécessaire (Freeman, 1979). Dobash et Dobash (1985) ont observé que la poursuite des démarches d'aide dépend souvent des réponses reçues et des effets perçus sur le comportement violent du conjoint (amélioration ou détérioration). Johnson (1985), dans une recension des écrits britanniques, note la similitude des attitudes et des réponses provenant des milieux médical, policier et de services sociaux : perception traditionnelle des femmes comme épouses et mères ; accent mis sur l'aspect privé du mariage et de la famille ; réticence à intervenir dans les cas de violence conjugale qui demeurent secondaires à leurs principales responsabilités professionnelles ; connaissance inadéquate du phénomène de la violence conjugale ; redéfinition en termes de soins aux enfants et intervention, lorsqu'elle a lieu, qui vise la réconciliation.

Parallèlement à ces observations, de nombreux changements sont survenus dans l'offre de services aux victimes de violence conjugale. Ce sont les femmes elles-mêmes qui ont d'abord compris l'urgence de mettre sur 
pied des ressources pouvant accueillir les femmes et leurs enfants. Ces premiers refuges ont levé le voile sur l'ampleur et la gravité des conséquences de la violence conjugale (Prud'homme, 1994). L'analyse féministe des causes et des fonctions sociales de la violence conjugale développée par ces groupes de femmes a permis de sortir ce problème de la sphère privêe et d'intervenir de façon globale dans une perspective radicalement différente de ce qui avait été fait jusque-là.

\section{L'ambivalence des victimes}

Le comportement de retrait des femmes violentées, en plein cœur d'une démarche entreprise pour échapper à la violence, est fréquent (Borkowski et al., 1983). Des femmes retournent avec leur conjoint après avoir séjourné dans une maison d'hébergement, d'autres abandonnent des démarches légales ou des relations d'aide. Les intervenants réagissent souvent par un mélange d'irritation et d'acceptation de cette ambivalence. Certains vont jusqu'à modifier leur pratique et deviennent plus hésitants et prudents dans l'offre d'aide. Cette attitude peut être ressentie par des clientes subséquentes et diminuer leur confiance dans la démarche entreprise, voire les décourager. Borkowski et al. (1983) affirment que les intervenants doivent comprendre la part que joue l'ambivalence dans les relations conjugales, même dans les circonstances les plus difficiles et dangereuses, pour éviter qu'ils ne développent cette hésitation à intervenir.

Les intervenants doivent également pouvoir apprécier la complexité des émotions vécues par les femmes, émotions qui se modifient au cours des étapes à franchir pour réorganiser leur vie et s'affranchir de la violence. Très souvent, les femmes vont chercher de l'aide au moment où elles vivent un grand stress, mais elles abandonnent leurs démarches lorsque la situation de crise se résorbe.

Turner et Shapiro (1986) analysent la rupture de la relation violente à la lumière du processus de deuil tel que décrit par Kübler-Ross (1969). Elles constatent que les intervenants s'attardent beaucoup aux services concrets et ne portent pas assez d'attention au puissant lien émotionnel qui empêche les femmes de réaliser une rupture permanente. En s'appuyant sur divers cadres théoriques, les auteures articulent les pertes associées à la rupture et elles décrivent les étapes du deuil qui mènent à la résolution de ces pertes.

\section{Le processus de recherche d'aide des femmes victimes de violence}

Nous présentons de façon succincte trois études qui ont cherché à mettre en évidence les éléments d'un processus de recherche d'aide propre aux femmes victimes de violence conjugale. Il s'agit des travaux 1) de Dobash et Dobash (1985), 2) de Limandri (1985) et 3) de Bilodeau (1987). 
1) Dobash et Dobash (1985) ont fait plusieurs constatations sur la base d'entrevues en profondeur avec 109 femmes. La recherche d'aide augmente avec le nombre d'incidents violents. Les proches sont plus susceptibles d'être contactés après la première attaque, et ce type de contact demeure fréquent dans le temps. Le type de contact se modifie à mesure que la violence continue et s'intensifie. Cette modification se manifeste par une augmentation substantielle des démarches entreprises auprès des agences formelles. Même dans le cas des attaques les plus violentes, les femmes sont plus susceptibles de rechercher de l'aide auprès de la famille et des proches avant de s'adresser aux professionnels.

Enfin Dobash et Dobash (1985) définissent quatre types de démarches classées par ordre croissant selon le degré de remise en question de la violence et de ses fondements sociaux et idéologiques : aide pour mettre fin à une attaque particulière; recherche d'une écoute ou d'un soutien moral, matériel ou médical après une attaque ; implication d'une tierce partie dans les négociations avec l'homme pour faire cesser la violence ; tentatives pour obtenir une aide matérielle (logement, aide financière, etc.) afin de sortir d'une relation violente.

2) Limandri (1985) a vérifié auprès de 40 femmes violentées l'influence de l'estime de soi, des conflits de rôle et du soutien social sur les modèles de recherche d'aide. L'analyse des données obtenues lui permet de mettre en évidence plusieurs paramètres de ce processus.

Elle aussi note que la première source de soutien provient des amis et de la famille et que, vers la fin de la relation, les femmes consultent davantage de professionnels. La recherche d'aide, d'abord motivée par un désir de changement dans la relation, s'oriente au fil des démarches vers l'aide permettant de sortir de la relation. À l'inverse des observations précédentes, Limandri observe une baisse de l'appui des amis et de la famille.

Pour Limandri, le processus de recherche d'aide se déploie dans le temps suivant un modèle non linéaire. La femme doit d'abord s'identifier comme étant une femme victime de violence. Cette auto-identification n'est pas nécessaire pour entreprendre un processus d'aide, mais elle est nécessaire pour obtenir de l'aide concernant l'abus proprement dit. Limandri distingue trois degrés d'ouverture de la part de la victime : l'ouverture complète, l'ouverture voilée ou l'invitation. La façon de rechercher de l'aide, active ou passive, est liée au type d'ouverture. La recherche active s'accompagne d'une ouverture complète sur la violence et de démarches directes pour obtenir de l'aide. Dans la recherche passive, la femme s'ouvre d'une façon voilée ou invitante dans un effort pour susciter l'aide. Le nombre d'aidants peut varier selon le nombre de personnes présentes dans le réseau social de la femme et le degré d'interrelations entre ce réseau et celui du partenaire. 
Plus leur estime de soi est faible, moins les femmes ont d'habiletés à s'affirmer et à rechercher l'aide des autres. Les stigmates associés à la recherche d'aide et au fait d'être abusée influencent la victime. La réponse de l'aidant à l'ouverture de la femme face à son vécu de violence affecte la continuité de sa démarche. Une réponse négative d'un aidant décourage la poursuite de l'aide auprès de cette source et même auprès d'autres sources. L'inverse est également vrai. La continuité du soutien de la part des sources d'aide formelle et informelle est un élément nécessaire à la poursuite de la recherche d'aide.

3) Bilodeau (1987) analyse en profondeur les démarches d'aide de 20 femmes qui ont séjourné en maison d'hébergement. Sur le plan théorique, elle s'appuie principalement sur deux modèles interactionnistes qui intègrent des variables socioculturelles et personnelles et accordent une grande importance aux possibilités présentes dans l'environnement.

Bilodeau considère la recherche d'aide comme un processus qui se construit dans le rapport de la femme aux réponses institutionnelles et communautaires présentes dans son environnement. La signification que les femmes donnent à leurs pratiques est considérée comme une réalité sociale distincte, une logique d'action qui fait appel aux valeurs, aux ressources, à la position sociale et aux responsabilités des femmes. L'analyse des résultats suggère que la recherche d'aide est enclenchée par une situation de crise ou par l'accumulation d'incidents de violence ou encore par une situation d'épuisement. Les femmes formulent peu de demandes explicites mais plutôt des appels à l'aide.

Bilodeau distingue trois types de démarches qui s'enchaînent et constituent les unités du processus de recherche d'aide. Le premier type de démarche se caractérise par la confidence et le soutien affectif et permet à la femme de composer avec la violence du conjoint. Il n'a donc pas d'effet sur la violence conjugale. Le deuxième type de démarche vise la protection immédiate et la récupération ou l'accumulation d'informations, de références auprès des ressources. Ces démarches peuvent mener à une période de rémission mais n'auront pas d'effets sur la violence si la femme n'utilise pas d'autres moyens. Le troisième type de démarche constitue une prise en charge par la femme de sa situation de victime de violence. Il peut s'agir de démarches qui favorisent le développement de l'autonomie et de l'affirmation de soi. On compte aussi le recours aux ressources pour marquer le refus de la violence, pour négocier un espace sans violence, ainsi que les démarches qui favorisent et permettent l'accomplissement de la rupture.

Bilodeau a identifié cinq séquences de démarches : les démarches visant à rendre tolérable la relation conjugale violente ; les démarches orientées vers le changement du conjoint; les démarches « d'autodéveloppement », 
orientées vers l'autonomie, l'affirmation et le refus de la violence; les démarches de confrontation pour forcer la négociation avec le conjoint; et finalement la rupture, la seule démarche, selon Bilodeau, qui produit la cessation de la violence, mais pas dans tous les cas puisque certaines femmes en subissent au-delà de la séparation. La femme utilise différemment les réponses de l'environnement au fur et à mesure qu'elle progresse à l'intérieur des séquences de démarches. Des événements particuliers interviennent dans le déroulement des séquences. Ainsi, les promesses non tenues du conjoint peuvent freiner le processus alors que les menaces de mort ou de blessures sérieuses peuvent l'accélérer.

\section{ÉTUDE D'UN CAS PARTICULIER : L'UTILISATION DE L'INTER- VENTION SOCIALE EN CLSC PAR DES FEMMES VICTIMES DE VIOLENCE CONJUGALE RÉFÉRÉES PAR LES POLICIERS}

L'étude maintenant présentée se situe dans le contexte général de la recherche d'aide des femmes victimes de violence conjugale et concerne plus particulièrement une expérience d'utilisation de deux types de ressources formelles qui ont conjugué leurs efforts : services policiers et services sociaux.

Devant la complexité du phénomène de la violence conjugale et la difficulté d'offrir un soutien social adéquat aux victimes, la direction du Service de police de la Communauté urbaine de Montréal (SPCUM) a recherché la collaboration du réseau des Centres locaux de services communautaires (CLSC). Le SPCUM (par le biais de 6 postes de police, soit les districts $32,34,42,45,53$ et 55) et quelques CLSC de la région métropolitaine (CLSC J.-Octave-Roussin, Montréal-Nord, Olivier-Guimond, Plateau Mont-Royal, Saint-Louis-du-Parc et Villeray, auxquels se sont ajoutés les CLSC Mercier-est/Anjou et Parc-Extension) ont entrepris, en janvier 1990, une expérience pilote de collaboration visant à offrir les alternatives psychosociales du CLSC aux femmes victimes de violence conjugale ayant fait appel aux policiers. Ces derniers suggèrent à la victime, entre autres possibilités, de la référer au CLSC afin qu'une intervenante entre en contact avec elle. Pour respecter les règles de confidentialité, l'autorisation écrite des victimes est requise. Dans un délai de 48 heures, les CLSC s'engagent à contacter les femmes signalées, à s'enquérir de leurs besoins et à entreprendre une démarche d'aide, s'il y a lieu. Ce projet pilote a été, depuis mai 1994, étendu à l'ensemble des CLSC et des postes de police de Montréal centre (Audy, 1994).

Comme on l'a vu précédemment, la recherche d'aide des femmes violentées auprès des ressources formelles est souvent enclenchée par une situation de crise ou par l'accumulation d'incidents de violence. De plus, 
l'utilisation d'une ressource formelle est associée au recours à d'autres ressources, tout en étant grandement tributaire de la réponse offerte par ces ressources. Les femmes doivent souvent s'adresser à plusieurs organismes pour obtenir toute l'aide nécessaire en raison de la diversité de leurs besoins et du manque de coordination entre les ressources. Le projet pilote de concertation entre le SPCUM et les CLSC apparaissait donc comme un moyen de favoriser la démarche d'aide des femmes violentées en leur offrant la possibilité de recevoir une aide psychosociale dans le contexte de crise provoquant l'intervention policière.

Une recherche exploratoire a été menée à l'intérieur de ce projet pilote afin d'identifier les caractéristiques distinguant les femmes violentées qui, à la suite d'un épisode de violence dans lequel les services policiers sont intervenus, consentent à ce que les CLSC entrent en contact avec elles et acceptent les services offerts, de celles qui refusent cette référence et ces services ${ }^{4}$. Cette étude voulait ainsi contribuer à une meilleure connaissance des conditions dans lesquelles les femmes victimes de violence conjugale utilisent les ressources formelles.

\section{MÉTHODOLOGIE}

La stratégie de recherche reposait sur un devis quantitatif de nature exploratoire où les données ont été recueillies par une méthode non obstructive. Cette approche a imposé des contraintes dans le choix des variables observées et a limité la quantité de caractéristiques étudiées. Par contre, cette approche était justifiée par l'état actuel des connaissances sur le processus de recherche d'aide des femmes victimes de violence conjugale qui permet d'identifier un certain nombre de facteurs afin de vérifier leur impact sur les décisions de la femme. Le devis adopté rendait possible la collecte d'un minimum d'informations sur plusieurs femmes participant au projet pilote CLSC/SPCUM, et permettait de généraliser les résultats à une autre expérience de même nature qui se déroulerait dans des milieux comparables.

Trois échantillons ont été construits. Le premier a été constitué à partir de tous les rapports d'événements de violence conjugale (1 005 rapports) enregistrés en 1991 dans les six districts de police qui ont participé au démarrage de l'expérience pilote. Le deuxième échantillon a été constitué, en principe, de toutes les victimes de violence conjugale référées par les policiers a huit CLSC (J.-Octave-Roussin, Mercier-Est/Anjou, Parc-Extension,

4. Cette étude a été réalisée grâce à une subvention de la Division de la prévention de la violence familiale de Santé et Bien-être Canada (maintenant Développement des ressources humaines Canada). Elle a, de plus, reçu un soutien financier de l'Université de Montréal et une contribution du Ministère du Solliciteur général du Canada. 
Montréal-Nord, Olivier-Guimond, Plateau Mont-Royal, Saint-Louis-du-Parc, Villeray) entre le $1^{\text {er }}$ octobre 1991 et le 31 décembre 1992. Au total, 177 grilles ont été remplies par les intervenantes et intervenants des CLSC participant à l'étude. Le troisième échantillon a été composé de femmes violentées s'adressant au CLSC d'elles-mêmes ou par un autre biais que celui de la transmission autorisée de leur nom au CLSC par les policiers. Nous avons obtenu l'information au sujet de 47 femmes, par la participation volontaire des intervenantes de six des huit CLSC concernés.

Dans le cadre de cet article, nous nous limitons à présenter les principaux résultats obtenus à partir du deuxième échantillon. Les données complètes de cette étude peuvent être consultées dans le rapport de recherche (Cantin et al., 1994).

\section{Instruments de collecte des données et variables retenues}

Les données ont été recueillies à partir de grilles d'information remplies par les intervenants ou intervenantes des CLSC participants. La nature des données recueillies a été identifiée à partir de consultations dans les CLSC et de l'expérimentation d'une première grille auprès des intervenantes consultées. Les variables suivantes ont ainsi été retenues : caractéristiques socio-démographiques (sexe, âge, scolarité, vie commune ou non au moment de l'agression, durée de vie commune, vie commune ou non au moment du contact par l'intervenante, lieu de résidence, occupation principale, nombre et âge des enfants vivant avec la victime, principale source de revenu); données sur l'agression (type d'agression, présence ou non de blessures, nature de ces blessures, soins médicaux, présence d'agressions antérieures et indication de leur fréquence, durée de la violence psychologique et physique, violence de la part de conjoints antérieurs, perception par la victime du fait que le problème est réglé ou non, sentiments de responsabilité par rapport à l'agression) ; utilisation antérieure des ressources (présence d'un réseau de parents ou d'amis, confidences faites à ces parents ou amis, utilisation antérieure de ressources formelles telles que policiers, maisons d'hébergement, services sociaux, médecins et avocats) ; intervention du CLSC (délai entre la référence par les policiers et le premier contact, acceptation ou non d'un rendez-vous par la victime, présence ou non à ce rendez-vous, type d'aide fournie, existence préalable d'un dossier au CLSC et connaissance ou non de la situation de violence).

\section{RÉSULTATS PROVENANT DE L'ÉCHANTILLON DES FEMMES RÉFÉRÉES PAR LES POLICIERS ET CONTACTÉES PAR LE CLSC}

Les femmes dont nous parlerons ici sont les victimes de violence conjugale dont les policiers ou policières ont fourni les coordonnées aux 
CLSC participant au projet conjoint CLSC/SPCUM entre le $1^{\text {tr }}$ octobre 1991 et le 31 décembre 1992 et pour lesquelles les intervenantes ont rempli une grille d'information, ce qui représente un échantillon de 177 femmes.

Parmi ces 177 femmes, 148 ont été rejointes par une intervenante et 29 n'ont pu l'être. Sur les 148 femmes qui ont été rejointes, 57 ont rencontré l'intervenante au moins une fois et 91 ne l'ont pas fait. La présentation des résultats mettra l'accent sur la comparaison entre les deux groupes. Les 57 femmes qui ont bénéficié d'au moins une rencontre au CLSC représentent $38,5 \%$ des 148 femmes rejointes par l'intervenante et $32,2 \%$ des 177 références.

\section{Caractéristiques socio-démographiques}

Il n'y a pas de différence significative, en ce qui a trait à la moyenne d'âge, entre les femmes qui acceptent de rencontrer une intervenante du CLSC $(32,96$ ans $)$ et celles qui refusent $(31,19 \text { ans })^{5}$. Les femmes qui vivaient avec leur conjoint au moment de l'agression sont représentées dans une proportion légèrement plus élevée $(89,3 \%$ contre $85,5 \%)$ parmi celles qui acceptent de rencontrer une intervenante du CLSC. En ce qui concerne le statut de vie commune actuelle (ou de vie commune antérieure) avec l'agresseur, les femmes qui acceptent de rencontrer une intervenante du CLSC sont plus nombreuses à être mariées (ou à avoir été mariées) que celles qui ne rencontrent pas l'intervenante.

Tableau 1

Répartition des femmes selon leur occupation principale

\begin{tabular}{|c|c|c|c|c|c|c|}
\hline \multirow[b]{3}{*}{ Occupation principale } & \multirow{2}{*}{\multicolumn{2}{|c|}{$\begin{array}{l}\text { Ensemble des } \\
\text { femmes }\end{array}$}} & \multicolumn{4}{|c|}{ Sous-échantillons $(\mathrm{N}=148)$} \\
\hline & & & \multicolumn{2}{|c|}{ Vont au CLSC } & \multicolumn{2}{|c|}{ N'y vont pas } \\
\hline & $\mathbf{N}$ & $\%$ & $\mathbf{N}$ & $\%$ & $\mathrm{~N}$ & $\%$ \\
\hline Au travail & 55 & 40,5 & 17 & 30,4 & 32 & 52,5 \\
\hline Au foyer & 54 & 39.7 & 23 & 41,0 & 23 & 37,7 \\
\hline En chômage ou invalidité & 6 & 4,4 & 2 & 3,6 & 2 & 3,3 \\
\hline Aux études & 15 & 11,0 & 11 & 19,6 & 2 & 3,3 \\
\hline En recherche d'emploi & 5 & 3,7 & 3 & 5,4 & 1 & 1,6 \\
\hline Autres & 1 & 0,7 & - & - & 1 & 1,6 \\
\hline Total & 136 & 100,0 & 56 & 100,0 & 61 & 100,0 \\
\hline Pas d'information & 41 & & 1 & & 30 & \\
\hline
\end{tabular}

5. $\mathrm{p} \leq 0,05$. 
Les femmes qui viennent au CLSC sont plus souvent des mères, ont une famille plus nombreuse et des enfants plus jeunes. Ces différences peuvent toutefois être apparues parce que l'information était davantage disponible pour les femmes qui ont rencontré une intervenante. Ces femmes sont plus nombreuses à être au foyer ou aux études (tableau 1) et plus nombreuses à avoir comme principale source de revenu les prestations d'aide sociale, comparativement à celles qui refusent de rencontrer une intervenante du CLSC (tableau 2). Les résultats de plusieurs études (MacLeod, 1987; Straus et Gelles, 1989 ; Rinfret-Raynor et Cantin, 1994) démontrent aussi qu'une grande proportion de femmes victimes de violence qui ont fait appel à des ressources formelles vivent sous le seuil de pauvreté. Rappelons toutefois que les femmes comptent parmi la population la plus pauvre au Canada, que la violence soit présente ou non au sein du couple. Les caractéristiques des femmes qui se sont présentées au CLSC s'apparentent à la description faite par Prud'homme (1994) du profil des femmes ayant séjourné dans une maison d'hébergement en 1990-1991. Prud'homme souligne une accentuation de la dépendance financière des femmes hébergées envers l'État depuis les cinq dernières années et une substitution de la dépendance financière visà-vis du conjoint par la dépendance envers l'État.

Tableau 2

Répartition des victimes selon leur principale source de revenu

\begin{tabular}{|c|c|c|c|c|c|c|}
\hline \multirow[b]{3}{*}{ Source de revenu } & \multirow{2}{*}{\multicolumn{2}{|c|}{$\begin{array}{l}\text { Ensemble des } \\
\text { femmes }\end{array}$}} & \multicolumn{4}{|c|}{ Sous-échantillons $(\mathrm{N}=148)$} \\
\hline & & & \multicolumn{2}{|c|}{ Vont au CLSC } & \multicolumn{2}{|c|}{ N'y vont pas } \\
\hline & $\mathbf{N}$ & $\%$ & $\mathbf{N}$ & $\%$ & $\mathbf{N}$ & $\%$ \\
\hline Son salaire & 32 & 26,9 & 12 & 21,1 & 16 & 34,0 \\
\hline Revenu du conjoint & 30 & 25,2 & 11 & 19,3 & 16 & 34,0 \\
\hline Assurance chômage & 8 & 6,7 & 4 & 7,0 & 3 & 6,4 \\
\hline Aide sociale & 31 & 26,1 & 21 & 36,8 & 6 & 12,8 \\
\hline \multicolumn{7}{|c|}{ Son salaire et autre source } \\
\hline de revenu & 13 & 10,9 & 5 & 8,8 & 6 & 12,8 \\
\hline Autres & 5 & 4,2 & 4 & 7,0 & - & - \\
\hline Total & 119 & 100,0 & 57 & 100,0 & 47 & 100,0 \\
\hline Pas d'information & 58 & & - & & 44 & \\
\hline
\end{tabular}

\section{Violence subie}

Lors de la prise de contact avec une intervenante du CLSC, près de la moitié des femmes $(46,9 \%)$ avaient subi des agressions physiques, et plusieurs avaient subi des agressions psychologiques $(24,3 \%)$. 
Les femmes qui acceptent de rencontrer une intervenante sont celles qui sont le plus représentées dans toutes les catégories d'agressions subies (tableau 3). Elles ont subi dans une plus grande proportion des gestes de violence physique tels que : recevoir des coups de pied, coups de poing ou être mordues ( $26,3 \%$ comparativement à $5,5 \%$ ) ; être poussées, agrippées, bousculées $(15,8 \%$ comparativement à $5,5 \%)$; recevoir une tape ou la fessée (10,5\% comparativement à $3,3 \%)$; être étranglées, étouffées $(10,5 \%$ comparativement à $3,3 \%$ ).

Tableau 3

Types d'agressions subies par les victimes

\begin{tabular}{|c|c|c|c|c|c|c|}
\hline \multirow[b]{3}{*}{ Types d'agressions subies } & \multirow{2}{*}{\multicolumn{2}{|c|}{$\begin{array}{l}\text { Ensemble des } \\
\text { femmes }\end{array}$}} & \multicolumn{4}{|c|}{ Sous-échantillons $(N=148)$} \\
\hline & & & \multicolumn{2}{|c|}{ Vont au CLSC } & \multicolumn{2}{|c|}{ N'y vont pas } \\
\hline & $N=177^{*}$ & $\%$ & $N=57^{*}$ & $\%$ & $N=91^{*}$ & $\%$ \\
\hline Agressions physiques & 83 & 46,9 & 45 & 78,9 & 31 & 34,1 \\
\hline \multicolumn{7}{|l|}{ Menaces d'agressions } \\
\hline physiques & 22 & 12,4 & 10 & 17,5 & 9 & 9,9 \\
\hline Menaces de mort & 22 & 12,4 & 13 & 22,8 & 6 & 6,6 \\
\hline Bris d'objets, dégâts & 1 & 0,6 & 1 & 1,8 & - & - \\
\hline \multicolumn{7}{|l|}{ Agressions psychologiques } \\
\hline précédente & 36 & 20,3 & 24 & 42,1 & 10 & 11,0 \\
\hline \multicolumn{7}{|l|}{ Agressions psychologiques } \\
\hline sans les catégories & & & & & & \\
\hline précédentes & 7 & 4,0 & 4 & 7,0 & 1 & 1,1 \\
\hline
\end{tabular}

* Il peut y avoir plus d'un type d'agression lors de l'incident.

Ces observations peuvent être dues à une meilleure connaissance de la situation par les intervenantes dans les cas où elles ont rencontré la femme. Il est cependant réaliste d'affirmer que plus la fréquence et la sévérité de la violence s'intensifient, pius ces femmes cherchent de l'aide auprès des ressources formelles. De plus, nous constatons que ces femmes sont plus nombreuses à avoir subi des agressions antérieures de la part du même conjoint ou ex-conjoint $(84,2 \%$ comparativement à $34,1 \%)$. Comme le soulignent plusieurs chercheures (Dobash et Dobash, 1985 ; MacLeod, 1987 ; RinfretRaynor et Cantin, 1994), il est rare que les femmes violentées qui s'adressent aux ressources formelles n'aient subi qu'une fois des actes de violence.

\section{Utilisation des ressources formelles et réseau de parents et d'amis}

Les femmes qui rencontrent une intervenante sont plus nombreuses à avoir fait appel à différentes ressources formelles (tableau 4). Encore ici, 
ces résultats peuvent être partiellement attribuables au peu d'information concernant les femmes qui ne se présentent pas au CLSC. Toutefois, le fait que les femmes qui ont consulté antérieurement différentes ressources soient moins hésitantes à l'égard de l'aide offerte par le CLSC rejoint l'hypothèse de Freeman (1979) et de Dobash et Dobash (1985) selon laquelle la méconnaissance des services offerts et l'inquiétude face à leurs réponses augmentent la réticence face à la recherche d'aide.

Tableau 4

Répartition des femmes selon les ressources utilisées

\begin{tabular}{lccccccc}
\hline & \multicolumn{2}{c}{$\begin{array}{c}\text { Ensemble des } \\
\text { femmes }\end{array}$} & \multicolumn{4}{c}{ Sous-échantillons $(\mathrm{N}=148)$} \\
Vont au CLSC & \multicolumn{2}{c}{$\mathrm{N}^{\prime} \mathrm{y}$ vont pas } \\
Autres ressources utilisées & $\mathrm{N}=177^{*}$ & $\%$ & $\mathrm{~N}=57^{*}$ & $\%$ & $\mathrm{~N}=91^{*}$ & $\%$ \\
\hline Maison d'hébergement & 11 & $\mathbf{6 , 2}$ & 7 & 12,3 & 3 & 3,3 \\
Médecin & 11 & $\mathbf{6 , 2}$ & 8 & 14,0 & 3 & 3,3 \\
Services sociaux & 14 & $\mathbf{7 , 9}$ & 8 & 14,0 & 5 & 5,5 \\
Police & 18 & 10,2 & 9 & 15,8 & 9 & 9,9 \\
Organismes d'entraide & 3 & 1,7 & - & - & 2 & 2,2 \\
Avocat & 5 & 2,8 & 3 & 5,3 & 1 & 1,1 \\
\hline
\end{tabular}

* Chaque femme peut avoir fait appel à plus d'une ressource.

Les deux tiers des femmes qui rencontrent une intervenante ont un réseau de parents ou d'amis et la moitié ont fait part de la violence subie à ces personnes. Ces données rejoignent l'hypothèse de Gourash (1978) et Kadushin (1969) à l'effet que la recherche d'aide auprès de professionnels survient après plusieurs démarches auprès de parents et d'amis.

\section{Perception de l'attitude de la victime}

Les femmes qui ne viennent pas au CLSC auraient davantage tendance à croire, selon la perception de l'intervenante, que le problème de violence ne risque pas de se reproduire $(64,1 \%$ contre $16,4 \%$ ). À l'inverse, les femmes qui désirent rencontrer une intervenante penseraient davantage que le problème n'est pas réglé $(69,6 \%$ contre $34 \%)$ ou que la violence risque de se reproduire $(14,3 \%$ contre $1,9 \%)$. Elles seraient aussi plus nombreuses à ne pas se sentir responsables des épisodes de violence, tandis que celles qui refusent de rencontrer une intervenante auraient au contraire davantage tendance à se sentir responsables des agressions. Soulignons cependant que l'information est manquante chez plus de la moitié de celles qui ne vont pas au CLSC. 


\section{Cohabitation agresseur et victime et lieu de résidence de la victime}

Il n'existe pas de différence entre les groupes en ce qui a trait à la cohabitation de l'agresseur et de la victime lors du premier contact avec une intervenante : $47,4 \%$ des femmes qui se présentent au CLSC et $47,5 \%$ de celles qui ne s'y présentent pas vivaient avec l'agresseur. Par contre, les femmes qui refusent de rencontrer une intervenante sont un peu plus nombreuses à habiter chez des parents $(9,2 \%$ comparativement à $5,3 \%$ chez celles qui vont au CLSC) ou amis $(6,6 \%$ contre $5,3 \%$ ) au moment ou elles ont été contactées. Évidemment, cette période de crise et de réorganisation de vie n'est pas propice à l'établissement d'une relation d'aide psychosociale, sans compter les facteurs liés au milieu de vie temporaire (par exemple, distance du CLSC).

\section{Prise de rendez-vous et type d'aide reçue au CLSC}

Les deux tiers des femmes référées par les policiers ont reçu une forme d'aide (de l'information surtout), mais, comme on l'a vu plus haut, seulement le tiers d'entre elles (57 sur 177) ont rencontré une intervenante. Il y aurait sûrement lieu d'analyser les éléments d'intervention qui conduisent à une plus grande efficacité dans ce processus de reaching out. Un élément dont il paraît essentiel de tenir compte est le délai entre la référence et la prise de contact. Les délais sont beaucoup plus courts chez les femmes qui se présentent au CLSC : une moyenne de 2,3 jours ouvrables comparativement à 3,4 chez celles qui ne vont pas au CLSC. Ce qui illustre à quel point la recherche d'aide est liée à la situation de crise lorsque la démarche de la femme vise la protection immédiate (un des types de démarches identifiés par Bilodeau, 1987).

Le fait de rencontrer l'intervenante est associé à une plus grande diversité des formes d'aide reçue, tant en ce qui concerne le suivi psychosocial et l'aide médicale que l'information et les références à d'autres ressources (tableau 5). De même, une proportion pius grande de femmes qui se présentent au CLSC ont été référées à des maisons d'hébergement $(7,0 \%: 4$ sur 57 comparativement à $2,2 \%: 2$ sur 91 ), à des ressources communautaires $(7,0 \%: 4$ sur 57 contre $4,4 \%: 4$ sur 91$)$, à l'aide juridique $(7,0 \%: 4$ sur $57)$, à des soins infirmiers $(5,3 \%: 3$ sur 57$)$, ou ont reçu de l'aide matérielle $(3,5 \%: 2$ sur 57$)$. L'accès à une ressource est donc, comme l'ont démontré plusieurs recherches, une porte d'entrée pour d'autres ressources.

\section{Dossier antérieur au CLSC}

Le tiers des femmes référées ( $58: 35,4 \%$ ) avaient déjà un dossier au CLSC (tableau 6). Les services déjà rendus à ces femmes sont surtout d'ordre 
médical et psychosocial. Dans un cas seulement sur quatre, la situation de violence était connue, et la majorité de ces femmes avaient reçu une intervention par rapport à cette situation.

\section{Tableau 5}

Répartition des femmes selon le type d'aide reçu au CLSC

\begin{tabular}{|c|c|c|c|c|c|c|}
\hline \multirow[b]{3}{*}{ Type d'aide reçue } & \multirow{2}{*}{\multicolumn{2}{|c|}{$\begin{array}{l}\text { Ensemble des } \\
\text { femmes }\end{array}$}} & \multicolumn{4}{|c|}{ Sous-échantillons $(N=148)$} \\
\hline & & & \multicolumn{2}{|c|}{ Vont au CLSC } & \multicolumn{2}{|c|}{ N'y vont pas } \\
\hline & $N=177^{*}$ & $\%$ & $N=57^{*}$ & $\%$ & $N=91$ & $\%$ \\
\hline Information & 104 & 58,8 & 50 & 87,8 & 41 & 45,1 \\
\hline \multicolumn{7}{|l|}{ Reférence à d'autres } \\
\hline ressounces & 51 & 28,8 & 33 & 57,9 & 10 & 11,0 \\
\hline Entrevues individuelles & 53 & 29,9 & 52 & 91,2 & - & - \\
\hline Suivi en groupe & 3 & 1,7 & 3 & 5,3 & - & - \\
\hline Aide médicale & 7 & 4,0 & 7 & 12,3 & - & - \\
\hline Autres & 7 & 4,0 & - & - & - & - \\
\hline
\end{tabular}

* Le total dépasse $100 \%$ car il pouvait y avoir plus d'un type d'aide reçu lors de la prise de contact.

\section{Tableau 6}

Répartition des femmes qui avaient un dossier au CLSC

\begin{tabular}{lrccccc}
\hline & \multicolumn{2}{c}{$\begin{array}{c}\text { Ensemble des } \\
\text { femmes }\end{array}$} & \multicolumn{4}{c}{$\begin{array}{c}\text { Sous-échantillons (N = 148) } \\
\text { Vont au CLSC }\end{array}$} \\
Dossier au CLSC & $\mathrm{N}$ & $\%$ & $\mathrm{~N}$ & $\%$ & $\mathrm{~N}$ & $\%$ \\
\hline Oui & 58 & 35,4 & 24 & 42,9 & 24 & 28,6 \\
Non & 106 & 64,6 & 32 & 57,1 & 60 & 71,4 \\
\hline Total & 164 & 100,0 & 56 & 100,0 & 84 & 100,0 \\
\hline Pas d'information & 13 & & 1 & & 7 & \\
\hline
\end{tabular}

Celles qui ont déjà un dossier sont davantage représentées parmi les femmes qui se présentent au CLSC et ces femmes sont plus nombreuses à avoir reçu des services médicaux et psychosociaux, comparativement à celles qui ne vont pas au CLSC. La violence conjugale était plus connue chez les femmes qui se présentent au CLSC (12,3\%: 7 sur 57 comparativement à 5,5\%: 5 sur 91$)$ et six d'entre elles $(10,5 \%: \mathrm{N}=57)$ avaient déjà reçu une intervention sur ce problème. Par ailleurs, parmi les cinq femmes déjà connues et qui ne se présentent pas au CLSC, quatre avaient déjà été suivies au sujet du problème de violence conjugale. Étant donné le faible nombre de sujets, ces différences doivent être interprétées avec prudence. 


\section{CONCLUSION}

Malgré les lacunes de cette étude, dues en grande partie à de nombreuses données manquantes qui ont limité les comparaisons entre les groupes de femmes, des observations intéressantes peuvent être réintroduites dans l'intervention sociale et policière, tandis que d'autres éléments suscitent des questions de recherche.

Sur le plan de l'intervention, les données confirment l'importance d'agir rapidement en situation de crise et de raccourcir le délai entre la référence policière et le contact par l'intervenante afin de favoriser l'acceptation de l'aide offerte par la femme. Des approches plus appropriées pourraient sans doute être développées pour favoriser le suivi en CLSC à la suite de la référence policière, puisque le tiers seulement des femmes réfêrées rencontreront une intervenante, et que près de la moitié de ces femmes y ont déjà un dossier. De plus, étant donné que les CLSC se distinguent entre eux dans leur capacité à faire accepter leur intervention par la femme violentée, on peut croire que des échanges entre les intervenantes sur les habiletés développées dans cette tâche et une formation pour développer ces habiletés contribueraient sans doute à améliorer l'efficacité de la démarche.

Comme nous l'avons déjà mentionné, d'un point de vue méthodologique, les différences obtenues entre les femmes qui utilisent les services du CLSC et les autres doivent être interprétées avec une grande prudence, à cause du nombre élevé de données manquantes sur plusieurs des variables étudiées. Les observations réalisées gagneraient donc à être vérifiées par la collecte directe d'informations auprès des femmes, plutôt que par le biais des intervenantes ou des dossiers.

Du point de vue conceptuel, notre étude nous permet d'affirmer que les éléments de la démarche de recherche d'aide des femmes violentées observés correspondent à ce que des travaux antérieurs nous avaient appris. Le recours aux ressources formelles est précédé ou accompagné du recours au réseau de soutien social constitué des parents et des amis. L'utilisation d'une ressource formelle favorise le recours à une autre ressource formelle, de telle sorte que les mêmes personnes reçoivent davantage d'aide et bénéficient d'une aide plus diversifiée.

\section{BIBLIOGRAPHIE}

AUDY, R. (1994), La collaboration entre policiers et intervenants sociaux : échec ou succès, Le travailleur social, vol. $62, \mathrm{n}^{\circ} 3: 3-136$.

BLODEAU, A. (1987), La Violence conjugale : recherche d'aide des femmes, Québec, Les Publications du Québec. 
BLAND, R. C., NEWMAN, S. C. et ORN, H. (1990), Health Care Utilization for Emotional Problems : Results from a Community Survey, Revue canadienne de psychiatrie, vol. 35, n' $5: 397-400$.

BORKOWSKI, M., MURCH, M. et WALKER, V. (1983), Marital Violence. The Community Response, Londres et New York, Tavistock.

CANTIN, S., RINFRET-RAYNOR, R. et FORTIN, L. (1994), Utilisation des ressources par les victimes de violence conjugale. Le cas des femmes référées par les policiers, Centre de recherche interdisciplinaire sur la violence familiale et la violence faite aux femmes, Collection Études et Analyses, $n^{\circ} 1$, Montréal, 114 pages.

CHENARD, L., CADRIN, H. et LOISELLE, J. (1990), État de santé des femmes et des enfants victimes de violence conjugale, Département de santé communautaire, Rimouski.

DePAULO, B. M. (1982), « Social-Psychological Processes in Informal Help-Seeking », dans WILLS, T. A., Basic Processes in Helping Relationships, New York, Academic Press : 255-279.

DePAULO, B. M. et FISHER, J. D. (1980), " The Costs of Asking for Help ", Basic and Applied Social Psychology, vol. $1: 23-35$.

DOBASH, R. E. et DOBASH, R. E. (1985), « The Contact Between Battered Women and Social and Medical Agencies", dans PAHL, J. (édit.), Private Violence and Public policy : The Needs of Battered Women and Responses of the Public Services, Londres et Boston, Routledge et Kegan Paul : 142-165.

FISHER, J. D. et NADLER, A. (1982), « Deterninants of Recipients Reactions to Aid : Donor-recipient Similarity and Perceived Dimensions of Problems m, dans WILLS, T. A. (édit.), Basic Processes in Helping Relationships, New York, Academic Press : 131-153.

FORD, D. A. (1983), «Wife Battery and Criminal Justice : A Study of Victim DecisionMaking ", Family Relations, vol. $32: 463-475$.

FREEMAN, M. D. A. (1979), Violence in the home, Saxon House, Teakfield Limited, Westmead, Fambotough, Hants, England.

GELLES, R. (1976), « Abused wives : Why do they stay ? ", Journal of Marriage and the Family, 38 : 659-668.

GOURASH, N. (1978), « Help-Seeking : A Review of the Literature ", American Joumal of Community Psychology, $6: 413-424$.

GOUVERNEMENT DU QUÉBEC (1995), Prévenir, dépister, contrer la violence conjugale, Politique d'intervention en matière de violence conjugale, ministères de la Santé et des Services sociaux, de la Justice, de la Sécurité publique, de l'Éducation et secrétariats à la Condition féminine et à la Famille, Québec.

GREENLEY, J. R. et MULLEN, J. A. (1990), Help Seeking and the Use of Mental Health Services, Research in Community and Mental Health, vol. $6: 325-350$.

GURIN, G., VEROFF, J. et FELD, S. (1960), American Views Their Mental Health, monographie 4, New York, Joint Commission on Mental Illness and Health, Basic Books.

HOTALING, G. T., FINKELHOR, D., KIRKPATRICK, J. T. et STRAUS, M.A. (1988), Coping with Family Violence, Newbury Park, Londres et New Delhi, Sage.

JOHNSON, N. (1985), Marital Violence, Londres et Boston, Routledge \& Kegan Paul.

KADUSHIN, C. (1969), Why people go to Psychiatrists, New York, Atherton.

KÜBLER-ROSS, E. (1969), On Death and Dying, New York, Macmillan Publishing. 
LIMANDRI, B. J. (1985), Help-Seeking Patterns of Abused Women: Self-Esteem, Role Conflict and Social Support as Influencing Factors, thèse de doctorat en « Nursing ", San Francisco, University of California.

MACLEOD, L. (1987), Pour de vraies amours... Prévenir la violence conjugale, Ottawa, Conseil consultatif canadien sur la situation de la femme.

MITCHELL, R. O., et HODSON, C. A. (1983), « Coping with Domestic Violence : Social Support and Psychological Health Among Battered Women ", American Journal of community, vol. $11, \mathrm{n}^{\circ} 6: 629-654$.

MOOS, R. H. et MITCHELL, R. E. (1982), « Social Network Resources and Adaptation : A Conceptual Framework, dans WILLS, T.A. (édit.), Basic Processes in Helping Relationships, New York, Academic Press.

PAHL, J. (1985), Private Violence and Public Policy: The Needs of Battered Women and Responses of the Public Services, Londres et Boston, Routledge et Kegan Paul.

PESCOSOLIDO, B. A. (1992), «Beyond Rational Choice : The Social Dynamics of How People Seek Help », American Journal of Sociology, vol. 97, $n^{\circ} 4: 1096-1138$.

PRUD'HOMME, D. (1994), "L'Impact des maisons d'hébergement sur les femmes violentées et leurs enfants ", dans RINFRET-RAYNOR, M. et CANTIN, S., La Violence conjugale. Recherches sur la violence faite aux femmes en milieu conjugal, Boucherville, Québec, Gaëtan Morin : 341-362.

RINFRET-RAYNOR, M. et CANTIN, S. (1994), « Violence conjugale rapportée par des femmes s'adressant aux services sociaux ", dans RINFRET-RAYNOR, M. et CANTIN, S., La Violence conjugale. Recherches sur la violence faite aux femmes en milieu conjugal, Boucherville, Québec, Gaëtan Morin : 3-22.

RINFRET-RAYNOR, M. et TURGEON, J. (1995), « Dépistage systématique de la violence conjugale. Réflexion théorique et développement d'un protocole ", Service social, vol. 44, n' $2: 57-90$.

RIOU, A., CHAMBERLAND, C. ET RINFRET-RAYNOR, M. (1996), « Conduites à caractère violent à l'endroit des conjointes », dans SANTÉ QUÉBEC ; LAVALLÉE, C., CLARKSON, M. et CHÉNARD, L. (dir.), Conduites à caractère violent dans la résolution de conflits entre proches, Monographie $n^{\circ} 2$, Enquête sociale et de santé 1992-1993, Montréal, ministère de la Santé et des Services Sociaux, gouvernement du Québec : 77-134.

RODGERS, K. (1994), La violence conjugale au Canada, Tendances sociales canadiennes, Automne 1994 : 3-9.

ROY, M. (1977), Battered Women, New York, Von Nostrand.

STRAUS, M. A. et GELLES, R. (1989), Physical Violence in American Families : Risk Factors and Adaptations to Violence in 8145 Families, Nouveau-Brunswick et Londres, Transaction.

TURNER, S. F. et SHAPIRO, C. H. (1986), « Battered Women : Mourning the Desth of Relationships ", Social Work, vol. 31, $\mathrm{n}^{\circ} 5: 372-376$.

VEROFF, J., DOUVAN, E. et KULKA, R. (1981), The Inner America, New York, Basic Books.

WALKER, L. E. (1979), The Battered Women, Harper \& Row, New York.

WARREN, D. I. (1981), Helping Networks. How People Cope with Problems in the Urban Community, Notre-Dame, Indiana, University of Notre Dame Press.

WILLS, T. A. (1982), Basic Processes in Helping Relationships, New York, Academic Press Inc., $521 \mathrm{p}$. 